Service social

\title{
Les femmes moins bien servies que les hommes ? Évaluation des services sociaux et de santé offerts aux minorités sexuelles
}

\section{Éric Alain Feugé, Line Chamberland, Olivia Kamgain et Jean Dumas}

Volume 63, numéro 2, 2017

Genres et sexualités chez les jeunes

URI : https://id.erudit.org/iderudit/1046502ar

DOI : https://doi.org/10.7202/1046502ar

Aller au sommaire du numéro

\section{Éditeur(s)}

École de travail social et de criminologie de l’Université Laval

ISSN

1708-1734 (numérique)

Découvrir la revue

Citer cet article

Feugé, É., Chamberland, L., Kamgain, O. \& Dumas, J. (2017). Les femmes moins bien servies que les hommes ? Évaluation des services sociaux et de santé offerts aux minorités sexuelles. Service social, 63(2), 99-113.

https://doi.org/10.7202/1046502ar
Résumé de l'article

Cet article s'appuie sur les données qualitatives issues d'une enquête en ligne menée auprès de 736 personnes LGBT résidant dans la région de Montréal et dans d'autres régions du Québec. Les résultats de cette enquête révèlent que les femmes se disent moins satisfaites que les hommes pour ce qui est de l'accès à des services sociaux et de santé adéquats. Les éléments jugés problématiques concernent la présomption de l'hétérosexualité, la divulgation de l'orientation sexuelle, l'inadéquation des services en santé sexuelle et l'inconfort des professionnel.le.s face à la non-conformité de genre. Le présent article met en lumière ces lacunes ainsi que les pistes suggérées pour rendre les services adéquats aux besoins des lesbiennes et des femmes bisexuelles. Il vient également enrichir le corpus de recherches sur les obstacles rencontrés par ces femmes dans l'accès aux services sociaux et de santé. 


\title{
Les femmes moins bien servies que les hommes? Évaluation des services sociaux et de santé offerts aux minorités sexuelles
}

\author{
FEUGÉ, Éric Alain, doctorant en psychologie \\ CHAMBERLAND, Line, Ph. D. sociologie \\ KAMGAIN, Olivia, M.A. administration publique \\ DUMAS, Jean, post-doctorant en communication
}

\section{RÉSUMÉ}

Cet article s'appuie sur les données qualitatives issues d'une enquête en ligne menée auprès de 736 personnes LGBT résidant dans la région de Montréal et dans d’autres régions du Québec. Les résultats de cette enquête révèlent que les femmes se disent moins satisfaites que les hommes pour ce qui est de l'accès à des services sociaux et de santé adéquats. Les éléments jugés problématiques concernent la présomption de l'hétérosexualité, la divulgation de l'orientation sexuelle, l'inadéquation des services en santé sexuelle et l'inconfort des professionnel.le.s face à la non-conformité de genre. Le présent article met en lumière ces lacunes ainsi que les pistes suggérées pour rendre les services adéquats aux besoins des lesbiennes et des femmes bisexuelles. II vient également enrichir le corpus de recherches sur les obstacles rencontrés par ces femmes dans l'accès aux services sociaux et de santé.

Mots-clés : Accès aux services de santé, minorités sexuelles, lesbiennes, bisexuelles, hétérosexisme

\begin{abstract}
This article is based on qualitative data collected in a survey of 736 LGBT people residing in the region of Montreal and other regions of the province of Quebec. This survey revealed that women report lower levels of satisfaction than men regarding access to adequate health and social services. Problematic aspects include the presumption of heterosexuality, the disclosure of sexual orientation, inadequacy of sexual health services and the discomfort of professionals with gender nonconformity. This article highlights these shortcomings as well as recommendations to make social and health services adequate to the needs of lesbian and bisexual women. This article also adds to research that focuses on the specific social and health services barriers faced by lesbian and bisexual women.
\end{abstract}

Key words: Access to health services, sexual minorities, lesbians, bisexual women, heterosexism 


\section{ENJEUX DE SANTÉ CHEZ LES MINORITÉS SEXUELLES}

Les écarts en matière de santé entre les minorités sexuelles et la population hétérosexuelle sont bien documentés dans la littérature scientifique. La prévalence élevée des infections transmissibles sexuellement et par le sang (ITSS) rapportée parmi les minorités sexuelles est souvent l'indicateur mis en avant pour illustrer les disparités en matière de santé. Or, on note également au sein des minorités sexuelles une prévalence élevée de maladies chroniques et de problèmes de santé mentale tels que l'anxiété et la dépression (Fredriksen-Goldsen et al., 2010 ; Tjepkema, 2008). Ces disparités en matière de santé seraient attribuables à la stigmatisation sociale et aux discriminations associées à l'appartenance à un groupe sexuel minoritaire (Chamberland et al., 2012). De plus, les personnes lesbiennes, gaies, bisexuelles et trans (LGBT) rencontrent plusieurs obstacles dans leur accès à des services sociaux et de santé adéquats, notamment des attitudes homophobes ou transphobes, des interventions inadéquates de la part des prestataires de services ainsi qu'un manque de connaissances, notamment médicales, au sujet des besoins et des réalités des personnes LGBT (Alencar Albuquerque et al., 2016). Par conséquent, les personnes LGBT sont plus susceptibles d'éviter les services sociaux et de santé ou de dissimuler leur orientation sexuelle ou leur parcours trans lorsqu'elles y ont recours.

Les rares recherches proposant une analyse différenciée des disparités en matière de santé entre les sous-groupes parmi les minorités sexuelles relèvent invariablement que les lesbiennes et les bisexuelles rencontrent des obstacles plus importants que les hommes de minorités sexuelles dans l'accès aux services (Alencar Albuquerque et al., 2016 ; Dumas, Chamberland et Kamgain, 2016 ; Tjepkema, 2008). Les études font également ressortir des écarts importants quant à l'accès à des services adéquats entre les personnes trans (dont l'identité de genre ne concorde pas avec le sexe qui leur a été assigné à la naissance) et les personnes cisgenres (pour lesquelles il y a concordance) (Alencar Albuquerque et al., 2016 ; Dumas et al., 2016 ; Bauer et al., 2015). Par ailleurs, relativement aux femmes hétérosexuelles, les lesbiennes et les bisexuelles rapportent un état de santé physique général moins bon, une prévalence plus élevée de problèmes de santé mentale, de tabagisme, d'alcool, mais aussi de surpoids et de stress (Fredriksen-Goldsen et al., 2010 ; Simoni et al., 2017), et ce, au Québec comme au Canada (Chamberland et al., 2012 ; Steele, Tinmouth et Lu, 2006). L'analyse secondaire des données de l'Enquête sur la santé dans les collectivités canadiennes (ESCC) (Tjepkema, 2008) montre que le taux de consultation d'un médecin de famille et les chances d'avoir reçu un test Pap (mesure de santé préventive du cancer du col utérin) sont plus faibles chez les lesbiennes (64\%) que chez les femmes hétérosexuelles $(77 \%)$, alors que, malgré la rareté des données sur ce type de cancer chez les lesbiennes, on sait que les risques de transmission du virus du papillome humain (VPH) entre femmes existent bel et bien, sans compter les divers partenaires sexuels au cours de leur vie. Enfin, une enquête québécoise sur l'accessibilité des services sociaux et de santé pour les lesbiennes a montré que des formes de lesbophobie, un manque de compétences des professionnel.le.s dans l'intervention auprès de ce groupe, mais aussi l'hétérosexisme intériorisé des lesbiennes elles-mêmes limitaient l'accès à des services adéquats (Mimeault, 2003).

Le présent article examine les perceptions des lesbiennes et des femmes bisexuelles au sujet de l'adéquation des services sociaux et de santé du Québec à leurs besoins et fournit des pistes pour améliorer la qualité des services. II vient ainsi actualiser les connaissances sur les obstacles à l'accès aux services sociaux et de santé rencontrés par les lesbiennes et les bisexuelles, et enrichir le corpus d'études qui s'intéressent à la santé des femmes de minorités sexuelles. 


\section{CAdRe THÉorique}

Selon Herek (2004), le concept d'hétérosexisme réfère à un système idéologique qui dénie, dénigre ou stigmatise toutes formes de comportement, d'identités, de relations et de communautés non hétérosexuelles. L'hétérosexisme définit l'ensemble des discours et des pratiques, individuels ou institutionnels, qui situent l'hétérosexualité comme la norme la plus acceptable socialement tout en infériorisant les autres sexualités (Chamberland, 2006). En raison du fait qu'il illustre le caractère systémique des attitudes négatives envers l'homosexualité et la nature structurelle des inégalités, le concept d'hétérosexisme est souvent privilégié par rapport à d'autres termes comme celui d'homophobie (Bastien Charlebois, 2011). De plus, ce concept permet de décrire des expériences de discrimination, mais aussi des pratiques professionnelles, des gestes et des propos quotidiens ayant en commun de dévaloriser et de dénier les corps et les vécus des lesbiennes et des bisexuelles, les maintenant ainsi dans le silence et l'invisibilité tout en soutenant la hiérarchie des orientations sexuelles. Le concept d'hétéronormativité s'avère lui aussi utile pour comprendre de telles expériences puisqu'il réfère aux discours qui construisent des normes concernant le sexe, le genre et l'orientation sexuelle, selon un idéal normatif de cohérence correspondant à la matrice sexe/genre/désir décrite par Judith Butler (2006). Cette matrice repose sur une construction binaire des catégories et l'alignement prescrit entre le sexe (mâle ou femelle), le genre (masculin ou féminin) et le désir nécessairement hétérosexuel, car basé sur la complémentarité entre les sexes/genres (Bastien Charlebois, 2011). Sont alors disqualifiés les corps, les expressions de genre, les sexualités et les identités se situant en dehors de cet idéal normatif.

Fish (2006) s'attache à préciser quelques mécanismes par lesquels l'hétérosexisme se maintient au sein du réseau des services sociaux et de santé. II y a tout d'abord la présomption systématique de l'hétérosexualité de la clientèle qui peut susciter une crainte de divulguer son orientation sexuelle (Fish, 2006). II y a également une injonction au silence qui se manifeste par des réactions négatives, réprobatrices à la suite de la divulgation d'une orientation autre qu'hétérosexuelle, par la censure ou par d'autres formes de disqualification de l'identité et de la vie privée de la personne. Au contraire, la manifestation d'une attitude positive vis-à-vis des personnes LGB de la part des professionnel.le.s de la santé entraîne une aisance à discuter de son orientation sexuelle et la divulgation de celle-ci est fortement associée à une utilisation régulière des services sociaux et de santé (Steele et al., 2006). La non-divulgation empêche le recueil d'informations nécessaires à une investigation psychomédicale appropriée et à l'obtention de services sociaux et de soins de santé adéquats (DeHart, 2008). De même, le confort ressenti par les personnes trans à discuter de questions de santé trans avec leur médecin de famille varie en fonction des expériences négatives antérieures et selon l'expertise dont le médecin fait preuve en matière de santé trans (Bauer et al., 2015). Enfin, il est important de mentionner que l'identité affichée d'une personne ne reflète pas nécessairement l'ensemble de ses comportements sexuels.

\section{MÉTHODOLOGIE}

Les données de cet article proviennent des résultats qualitatifs d'une enquête en ligne menée de janvier 2012 à novembre 2013 auprès des usager.ère.s LGBT du réseau québécois de la santé et des services sociaux, dans le cadre d'une recherche-action participative menée en collaboration avec le CSSS Jeanne-Mance (Dumas et al., 2016). Cette recherche a été financée par le Programme 4P (Promotion, Prévention et Politiques publiques) du Réseau de recherche en santé des populations du Québec, sous la forme d'une bourse postdoctorale au chercheur principal (Jean Dumas). Le projet de 
recherche a reçu l'approbation du Comité institutionnel d'éthique de la recherche avec des êtres humains de l'UQAM en septembre 2011 (certificat $n^{\circ}$ 701005).

Le recrutement de participant.e.s a été effectué par Internet avec la collaboration de plus de 80 organismes communautaires offrant des services aux personnes LGBT. Des affiches et des cartes d'invitation ont également été distribuées lors de la rencontre d'usager.ère.s des services du CSSS Jeanne-Mance, aux membres et usager.ère.s de plusieurs organismes LGBT, et diffusées dans des magazines, dans divers lieux du Village gai de Montréal et dans des institutions d'enseignement supérieur (cégeps et universités). L'échantillon final comprend 736 questionnaires et est constitué de $50,3 \%$ de femmes (370) et 49,7\%, d'hommes (366). Il est à noter que 8,7\% de l'ensemble des participant.e.s se sont identifiés comme trans (transgenres, transsexuel.le.s, travestis ou ayant un passé de personne trans) et 1,9\% comme personnes intersexuées. La moyenne d'âge de l'ensemble des répondant.e.s est de 36 ans et un peu plus des deux tiers des répondant.e.s résident dans la région de Montréal $(n=477)$. En ce qui concerne l'orientation sexuelle autodéfinie, près des deux tiers des femmes se considèrent comme homosexuelles ou lesbiennes et près des trois quarts des hommes comme homosexuels ou gais. Sur l'ensemble des répondant.e.s, $13,9 \%$ se considèrent comme bisexuel.le.s et 6,7\% comme queers. Les personnes trans sont plus nombreuses que les personnes non trans à s'identifier comme bisexuelles ou queers. Dans cet article, nous nous intéressons aux femmes cisgenres se déclarant lesbiennes ou bisexuelles, mais également à celles qui s'identifient comme queers ou trans.

Pour la collecte de données, le questionnaire mesurant la satisfaction des usager.ère.s à l'égard des Centres de santé et de services sociaux au Québec dans le cadre du processus d'agrément (ou démarche d'assurance de la qualité) a été repris intégralement et enrichi par l'ajout de 11 affirmations référant à des dimensions propres aux réalités des personnes LGBT, telles que l'aisance à discuter de son orientation sexuelle ou de son identité de genre avec un.e professionnel.le de la santé et la perception que les services sont adaptés aux besoins des personnes LGBT (Dumas et al., 2016). Le questionnaire portait également sur les habitudes d'utilisation des services sociaux et de santé (types de services, fréquence, motifs). Trois sections du questionnaire offraient la possibilité aux participant.e.s de décrire ou commenter des situations vécues relativement à l'évitement des services, à l'aisance ou au malaise à discuter de son orientation sexuelle ou de son parcours trans et à la qualité des interactions avec les prestataires de soins. Au total, 2175 commentaires ont été recueillis dont 1069 ont été rapportés par les hommes, 1106 par les femmes. Les réponses ouvertes du questionnaire ont fait l'objet d'une analyse de contenu thématique à l'aide du logiciel NVivo (QSR, version 10, 2012) réalisée conjointement par l'auteur principal et deux assistant.e.s de recherche, ce qui a permis d'assurer un accord inter-juges lors de la codification.

\section{PRÉsentation des RÉsultats}

La présentation des résultats s'articule autour des quatre catégories thématiques dégagées de l'analyse, soit la présomption de l'hétérosexualité, les facteurs favorisant ou compromettant l'aisance à discuter de l'orientation sexuelle ou de l'identité de genre, l'inadéquation des services de dépistage et de prévention des ITSS auprès des lesbiennes et des bisexuelles, et l'inconfort des professionnel.le.S face à une expression de genre non conforme aux normes hétérosexistes. Les principales recommandations préconisées par les participantes seront ensuite exposées. 


\section{La présomption de l'hétérosexualité}

Les répondantes se sont fortement exprimées sur la pression ressentie à se conformer à un modèle hétérosexuel lors de leurs consultations avec des professionnel.le.s de la santé et des services sociaux. Certain.e.s prestataires de services présumeraient d'emblée l'orientation hétérosexuelle des usagères dans les questions qu'ils et elles posent lors des consultations, notamment en matière de santé sexuelle : "Trop souvent les médecins ou les infirmières, en dehors du CLSC, présument que mon partenaire est un homme et que je n'en ai qu'un seul. II faut que je leur explique pourquoi je ne me protège pas durant les relations sexuelles (lesbienne, 26 ans). » Une femme a rapporté s'être sentie étiquetée par l'attribution d'une orientation autre qu'hétérosexuelle: «Pour le dépistage, je me suis sentie très jugée dans mes comportements et mon orientation [...] parce que je n'avais pas coché "bisexuelle" sur le questionnaire d'accueil, mais mentionné avoir eu des rapports avec des femmes. La médecin m'a regardée en me disant: "C'est ça, la bisexualité !" Hum, zéro professionnalisme (Bisexuelle, 28 ans). » Ce dernier exemple fait exception. II apparaît que les participantes ont très majoritairement rapporté des expériences de présomption d'hétérosexualité, notamment à l'occasion d'une consultation avec leur médecin de famille. Les propos rapportés ci-dessous témoignent de la forte contrainte à l'hétérosexualité que peuvent exercer certain.e.s prestataires de services. Souvent, leurs préconceptions quant aux comportements sexuels des répondantes dissuadent ces dernières de se montrer franches à ce propos, malgré la présence d'indices ostensibles :

Quand elle [ma médecin de famille] me demande si je suis active sexuellement, elle prend pour acquis que c'est une relation hétérosexuelle (même si je ne donne aucune information sur ma partenaire) et je me sens mal à l'aise de la contredire. (Lesbienne, 31 ans)

Elle [ma médecin de famille] m'a demandé si j'avais un chum. J'ai dit non. Quand j'ai sorti mon agenda devant elle, avec plusieurs collants du Gay-flag et des gigas «NON À L'HOMOPHOBIE » dessus, elle n'a même pas compris et a continué à me parler de chum... Ça m'a coupé l'envie de lui partager mes problèmes d'orientation sexuelle. (Lesbienne, 21 ans)

Plusieurs travailleurs sociaux ne sont pas à l'aise de questionner sur l'orientation sexuelle des personnes qu'ils rencontrent. Certains intervenants prennent pour acquis que la personne est hétérosexuelle. Je me suis butée par le passé à ce même genre de questions. (Lesbienne, 22 ans)

Certaines répondantes n'ayant pas abordé la question de leur orientation sexuelle lors d'une consultation médicale ont déploré la réaction d'étonnement, voire de consternation, des prestataires de services lorsqu'ils ou elles apprenaient que leur patiente n'utilisait pas de moyen contraceptif. Ainsi, le questionnement quant au mode de contraception utilisé par les usagères s'associe également à une présomption de l'hétérosexualité : «Quel contraceptif j'utilise ? La réponse est aucun. La réaction des infirmières est l'étonnement. Je leur dis alors que ma partenaire est une femme... [...] Je ne sais pas si c'est parce que "j'ai pas l'air lesbienne", mais en général, je sens les infirmières démunies devant la situation (lesbienne, 28 ans). " Finalement, plusieurs répondantes ont dénoncé la projection implicite de normes ou l'imposition du modèle traditionnel à leur égard voulant que la famille soit nucléaire et le couple, monogame. L'une d'entre elles se désole de constater que la famille homoparentale est parfois perçue comme une famille de seconde classe, n'étant pas encore reconnue comme une cellule 
familiale viable : «Ne pas penser que tous les enfants viennent avec un papa et une maman. II y a plusieurs types de familles. À la visite de l'hôpital pour la naissance du bébé, l'infirmière ne cessait de répéter : "le papa, le papa, le papa" (Lesbienne, 29 ans). ”

\section{Facteurs favorisant ou compromettant l'aisance à discuter de l'orientation sexuelle}

La majorité des femmes ont indiqué se sentir très à l'aise ou à l'aise de discuter de leur orientation sexuelle avec un.e professionnel.le de la santé ou des services sociaux. Le principal facteur favorisant cette aisance rapportée par les répondantes, plus souvent que par les hommes de l'étude, est une pleine acceptation et une intégration totale de leur orientation sexuelle au sein de leur identité. La citation qui suit illustre cette affirmation inconditionnelle de leur orientation sexuelle : "Toutes les personnes que je côtoie connaissent mon orientation sexuelle et je ne sens le besoin de la cacher dans aucun contexte (lesbienne, 27 ans). " Les participant.e.s à l'étude, quelle que soit leur identité de genre (homme/femme cisgenre ou trans), étaient plus enclin.e.s à aborder la question de leur orientation sexuelle lorsqu'ils ou elles percevaient une certaine réceptivité de la part de leur interlocuteur.trice, une attitude d'ouverture à la diversité des pratiques sexuelles, de non-jugement par rapport à l'orientation sexuelle, ainsi que le respect et l'écoute vis-à-vis des usager.ère.s.

J'ai eu un suivi à long terme avec une travailleuse sociale très ouverte qui m'a beaucoup aidée à accepter mon orientation sexuelle. (Femme queer, 27 ans)

À plusieurs occasions, j'ai senti une attitude ouverte par rapport à la sexualité en général, incluant l'orientation sexuelle. Je me suis sentie reçue comme une personne d'abord, mes préférences sexuelles n'étant pas vues comme un élément choquant ou surprenant. (Femme queer, 30 ans)

Contrairement aux hommes ayant participé à l'enquête, les femmes ont rarement avancé que la similarité de sexe/genre ou d'orientation sexuelle (p. ex. patient et médecin gais), réelle ou perçue, entre elles-mêmes et la personne offrant le service contribuait à leur aisance à dévoiler ou à discuter de leur propre orientation sexuelle. Certaines répondantes qui rapportaient ne pas être à l'aise de discuter de leur orientation sexuelle avec un.e professionnel.le ont déclaré être mal à l'aise avec l'ensemble des personnes qui ne leur sont pas familières ou bien "être dans le placard " dans la plupart de leurs milieux de vie. Comme l'illustre le témoignage ci-dessous, certaines participantes expliquent également leur malaise par une certaine incertitude ou une difficulté à classifier leur orientation sexuelle selon des catégories prédéfinies.

J'étais légèrement mal à l'aise à cause de mon manque d'habitude de parler de mon orientation sexuelle, mais également à cause de ma difficulté à définir mon orientation sexuelle en fonction des standards hétérosexuels/homosexuels. Cette difficulté à me définir et à exprimer clairement ce que je ressens, ce que je désire et comment je me perçois contribuait également à accentuer ce malaise. (Femme trans, lesbienne, 35 ans)

La crainte d'être jugée, la peur d'être rejetée ou traitée différemment ont été plus souvent rapportées par les femmes que par les hommes parmi les raisons justifiant le malaise à discuter de leur orientation sexuelle ou de leur parcours trans avec un prestataire de services. Plusieurs répondantes craignent un 
changement d'attitude de la part de ce dernier ou une moindre qualité des services à la suite d'une telle divulgation.

On ne sait jamais comment l'autre va prendre ça! Elle ou il a-t-elle/il des préjugés ? Dans une situation de rencontre médicale, je peux facilement dire que je suis moins forte et donc moins capable de défendre tel ou tel point. (Femme en questionnement, 51 ans)

Faire son coming out à un professionnel de la santé n'assure pas qu'il va t'accepter et qu'il ne va pas essayer de ramener tous tes problèmes à ton homosexualité en essayant de te dire que c'est ça le problème et qu'il faut changer. [...] C'est donc stressant de faire un coming out à un professionnel. (Femme trans, lesbienne, 29 ans)

Parmi les différents intervenant.e.s professionnel.le.s, c'est le médecin qui suscite le plus cette inquiétude, sans doute à cause de la connotation d'autorité qui se rattache à ce dernier. Cette appréhension de la réaction des professionnel.le.s à la suite d'une divulgation de l'orientation sexuelle peut amener certaines femmes à taire leur orientation ou même à restreindre leur utilisation des services : « J'évite les consultations gynécologiques, de un par peur, mais de deux parce que je n'ai pas envie de dévoiler mon orientation sexuelle à un inconnu (lesbienne, 21 ans). " II en va de même pour l'identité de genre, comme l'expose cette répondante: «Après une mauvaise expérience, je me sens mal à l'aise avec les services psychosociaux et je consulte sans dévoiler mon orientation ou mon genre réel (Femme queer, 20 ans). " Finalement, de nombreuses participantes lesbiennes et bisexuelles, particulièrement dans les milieux ruraux, ont déclaré qu'il était parfois difficile de dévoiler leur orientation sexuelle à leur médecin de famille, puisqu'il traite ou connaît également d'autres personnes de leur entourage proche.

\section{L'inadéquation des services de dépistage et de prévention des ITSS}

Plusieurs répondantes rapportent un manque de connaissances de la part des prestataires de soins en ce qui a trait aux pratiques sexuelles des femmes lesbiennes, bisexuelles, queers ou trans. La minimisation des risques de transmission des ITSS est l'élément le plus fréquemment rapporté. L'idée d'une «immunité » des lesbiennes face aux ITSS ferait partie des représentations largement répandues, y compris chez les premières concernées.

Souvent, on banalise les risques de transmission des ITSS et on ne parle pas des façons de se protéger. II m'est déjà arrivé de dire que j'étais lesbienne et le médecin a laissé tomber son questionnaire [...] sans me poser de questions à propos de mes comportements en matière de sexualité. (Bisexuelle, 26 ans)

À aucun moment, on ne m'a expliqué qu'il y avait aussi des moyens de se protéger durant les relations avec des femmes. À ce jour, je ne suis pas plus éclairée sur le sujet. (Lesbienne, 35 ans)

De plus, certaines répondantes déplorent que les campagnes de prévention des ITSS ciblent uniquement les personnes ayant des relations sexuelles avec des hommes. Elles préconisent que soit disponible l'information au sujet des ITSS autres que le VIH ainsi que sur les pratiques sécuritaires autres que le port du condom. 
Plusieurs jeunes lesbiennes de ma communauté sont persuadées qu'entre filles, il n'y a aucun risque et que s'il arrive d'avoir des ITSS chez les lesbiennes, ce serait à cause des bisexuelles. En plus d'accentuer la biphobie, le manque d'information amène les lesbiennes à prendre des risques qui pourraient être facilement évités. (Lesbienne, 22 ans)

En fait, à l'heure actuelle, beaucoup trop [de personnes] ont enregistré le message [que] « safer sex $=$ condoms » et par conséquent le message inverse «ITSS = pénétration à découvert ». [Donc] tout ce qui n'inclut pas un pénis dans un vagin devient une pratique safe selon les jeunes. (Lesbienne, 22 ans)

Certaines répondantes expriment par ailleurs leur insatisfaction quant à l'accessibilité effective de moyens de protection contre les ITSS, notamment les digues dentaires, et quant à la qualité de celles fabriquées maison.

Considérant que la protection sexuelle disponible pour les cunnilingus ne se retrouve que dans les sex shop, [...] et que par conséquent les mineurEs n'y ont pas accès, il me semble essentiel de fournir des digues dentaires dans les cliniques jeunesse, les pharmacies et les écoles secondaires comme il se fait déjà pour les condoms. (Lesbienne, 28 ans)

[À propos des digues dentaires fabriquées à partir de condoms] Tous les condoms offerts dans les écoles et cliniques jeunesse sont lubrifiés (donc turn off pour le cunnilingus et aussi pour la fellation). Et de plus, si les jeunes ont de la difficulté à utiliser le condom conventionnellement, imagine le truc quand il faut que tu prennes les ciseaux pour en faire un rectangle. (Lesbienne, 22 ans)

Enfin, plusieurs répondantes déplorent que les tests Pap ne leur soient pas proposés au même titre qu'aux femmes hétérosexuelles: "My physician dismissed the need to give me a Pap smear when I told her I had only had sex with women (Bisexuelle, 23 ans). " Que la décision médicale soit justifiée ou non importe moins ici que le manque de confiance de nombreuses femmes envers l'expertise médicale, de même que les doutes concernant la lecture qui est faite des risques d'ITSS encourus à partir des informations, souvent incomplètes, livrées dans le contexte de la consultation ou des conclusions qui en sont erronément déduites quant à leurs comportements sexuels actuels ou antérieurs.

\section{Inconfort des professionnel.le.s face à une expression de genre non traditionnelle}

Dans notre étude, de nombreuses personnes s'identifiant comme queers ont dénoncé le déni de leur identité par les professionnel.le.s de la santé et des services sociaux, que ce soit relativement à leur expérience intime du genre ou à l'expression de leur genre.

I feel that I am never given the option to define my own gender. Rather, I have to check a box of male or female. Being genderqueer, this bothers me to no end. I want to see professionals who will treat me based on my own given identity, rather than how they read my gender visually. (Femme trans, queer, 30 ans)

Dans les commentaires ajoutés par les répondant.e.s au questionnaire d'enquête, des expériences négatives en lien avec leur aspect corporel ont été rapportées exclusivement par des femmes, de 
diverses orientations sexuelles et identités de genre, de même que par des hommes trans (qui ne font pas l'objet de cet article). Par contre, on ne relève aucun propos de ce type venant des hommes cisgenres. Les répondantes ont été particulièrement volubiles quant à l'inconfort éprouvé par certains prestataires de services lorsque l'apparence de leur corps ne correspond pas au modèle traditionnel de la féminité, notamment en ce qui a trait à la pilosité. Cet inconfort des professionnel.le.s de la santé face à certains aspects de leur corps met évidemment en péril le lien de confiance entre elles et les prestataires de services, et peut avoir une incidence sur la motivation de certaines à utiliser à nouveau les services.

In my experience, almost all the male doctors I have seen in walk-in clinics [...] have acted very uncomfortably in response to my sexual identity and history, and/or to my body (my leg and arm pit hair). It makes me miserable. I want to be able to trust these people, and they clearly hate or dislike my body. It's a horrible feeling, because I am depending on them for care. (Bisexuelle, 30 ans)

Les données recueillies ne permettent pas de savoir si la fréquence de telles expériences varie selon le sexe/genre du prestataire de services. Quelques commentaires soulignent par ailleurs la méconnaissance de la diversité des parcours trans, notamment ceux qui diffèrent du modèle traditionnel de la transsexualité impliquant une transition médicale, et l'incapacité qui en découle d'apporter une aide appropriée à la personne qui consulte. Rappelons-le : l'expérience de son genre par une personne est subjective, celle-ci peut s'identifier comme homme ou femme ou utiliser d'autres termes, et ce, indépendamment de son sexe biologique, et ce parcours identitaire ne s'accompagne pas nécessairement du désir de modifier son corps. Cette vision peine à s'intégrer dans les approches d'intervention de certain.e.s professionnel.le.s qui peuvent aller jusqu'à dissuader les usagères de consulter.

Quand j'ai consulté la travailleuse sociale [...] et que j'ai discuté de mon questionnement sur le genre, elle m'a posé plusieurs questions sur la manière dont je me sens par rapport à mon corps et à mes organes génitaux. Étant à l'aise avec mes organes génitaux et l'ayant exprimé, elle a fini par me dire : «Ben, c'est quoi le problème, d'abord ? » J'ai l'impression qu'il existe une sorte de "trans narrative» dans le milieu de la santé et des services sociaux. Les personnes qui cadrent dans cette narration ont déjà de la difficulté à obtenir des services... mais quand on ne cadre pas dedans, c'est franchement pénible. [...] La travailleuse sociale a refusé de continuer à travailler avec moi et m'a invitée à ne revenir que si j'avais des problèmes «plus sérieux » à régler. Ça a été très dur pour moi. (Femme queer, 24 ans)

\section{Pistes D'intervention}

Les répondantes ont formulé plusieurs suggestions qui pourraient améliorer la qualité et l'adaptation des services sociaux et de santé. Leurs idées ont été regroupées autour de deux pistes d'intervention : la sensibilisation des prestataires des services sociaux et de santé et l'adaptation des services à des besoins spécifiques. 


\section{Sensibiliser les prestataires des services sociaux et de santé}

Au vu du grand nombre de commentaires énoncés par les répondantes sur ce sujet, les prestataires des services sociaux et de santé ont un rôle déterminant à jouer pour assurer une meilleure adéquation des services aux besoins des lesbiennes et des bisexuelles. Près du quart des suggestions émises concernent la sensibilisation des intervenant.e.s aux réalités des personnes LGBT, durant leur formation collégiale ou universitaire, ou par le biais de formations en milieu du travail. II ressort de ces suggestions que quatre éléments en particulier devraient faire partie d'une telle conscientisation pour enrayer les méfaits de l'hétérosexisme :

1. La non-présomption du sexe/genre du patient et de son/sa partenaire : "Please caution health workers (nurses, doctors, orderlies, anyone) AGAINST assuming the sex of someone's partner. Heteronormativity sucks (lesbienne, 21 ans). "

2. L'utilisation d'un vocabulaire non genré : «Former le personnel sur l'importance d'adopter un vocabulaire inclusif et sur les réalités des personnes LGBT (bisexuelle, 36 ans). ”

3. La conscientisation aux réalités et aux pratiques sexuelles des personnes LGBTQ et sur les risques de transmission d'ITSS lors de relations sexuelles entre femmes ou avec des partenaires de tous genres, afin que les intervenant.e.s puissent répondre adéquatement à leurs questionnements sur leur santé sexuelle et promouvoir des pratiques sécuritaires.

J'ai assisté à une formation sur l'intervention sur l'homosexualité avec plusieurs employéEs de milieux communautaires et CSSS. En général, la formation était très utile pour apprendre des éléments de base sur [l'homosexualité]. Par exemple, certaines personnes ignoraient absolument tout sur la vie sexuelle. Une formation de ce genre est très utile pour tous les employéEs. (Lesbienne, 25 ans)

4. L'inclusion d'un volet de lutte contre l'homophobie et les discriminations au sens large dans les programmes de formation : "Des formations auprès des professionnel.le.s de la santé devraient être largement offertes pour les sensibiliser à l'homophobie (gayphobie, lesbophobie, transphobie, et intersexualité), les informer sur les ressources LGBT offertes au Québec, nos réalités (santé, psychologie, discriminations vécues...). (Femme en questionnement, 27 ans)

Enfin, des répondantes suggèrent que le travail de proximité et la formation dispensée par des personnes directement concernées par les problématiques d'accès aux services liées à l'orientation sexuelle et à l'expression de genre pourraient grandement bénéficier à la conscientisation des prestataires de services par rapport aux enjeux qui affectent les personnes LGBTQ.

\section{Adaptation des services aux besoins spécifiques}

Des répondantes suggèrent d'inclure les réalités lesbiennes et bisexuelles dans les actions et campagnes de prévention, notamment en matière de santé sexuelle, et de rendre disponibles des informations justes et appropriées sur les ITSS. Il est également proposé d'accroître les signes visibles d'acceptation et d'ouverture à l'égard des personnes LGBT dans les lieux où se donnent des services sociaux et de santé afin de démontrer la volonté de l'organisation d'offrir des services adéquats aux personnes de minorités sexuelles: "Une affiche dans une salle d'attente qui montre qu'il y a une ouverture pour les personnes homosexuelles met en confiance (lesbienne, nd). » 


\section{Discussion}

Cet article se base sur les résultats qualitatifs d'une enquête qui a mis en évidence que les personnes LGBT ont globalement un taux de satisfaction élevé à l'égard des services sociaux et de santé au Québec, à l'exception des personnes trans qui se montrent insatisfaites à plusieurs égards (Dumas et al., 2016). Selon les données quantitatives de l'enquête, les hommes et les femmes ne se distinguent pas quant à certains indicateurs de satisfaction générale à l'égard des services ( $p$. ex. qualité de l'accueil, respect et politesse), mais les femmes apparaissent moins satisfaites que les hommes par rapport à plusieurs aspects qui concernent l'adéquation des services à leurs besoins et réalités propres, à savoir : la présomption de l'orientation sexuelle, l'écoute et la compétence des intervenant.e.s, leur aisance à discuter de l'orientation sexuelle ou de l'identité de genre, leur compréhension de la situation de l'usager.ère et l'utilisation de termes appropriés pour en discuter, ainsi que l'amélioration apportée par les services reçus (Dumas et al., 2016). Dans l'ensemble, l'analyse des commentaires écrits en réponse à l'invitation faite aux répondant.e.s de décrire ou qualifier des situations vécues va dans la même direction. Il en ressort que les femmes, qu'elles se définissent elles-mêmes comme lesbiennes, bisexuelles, queers ou trans, se considèrent globalement comme moins bien servies, alors que les hommes se disent plus satisfaits des services reçus. Nous discuterons ici de trois hypothèses explicatives de cette distinction assez nette entre les hommes et les femmes quant à l'adaptation des services à leurs besoins.

La première hypothèse renvoie aux discours et pratiques hétérosexistes dans les institutions offrant des services de santé. Ici, la présomption de l'hétérosexualité va de pair avec des représentations erronées de la sexualité des lesbiennes et des bisexuelles, représentations qui délégitiment leurs relations affectives et sexuelles. L'idée subsiste, notamment chez les professionnel.le.s de la santé et des services sociaux, d'une sexualité lesbienne qui n'implique pas de pénétration ou de coït vaginal, ou qui est interprétée comme une non-sexualité. Parallèlement, il existe peu d'espaces de représentation mettant en scène des modèles variés de la sexualité lesbienne hors du voyeurisme et des fantasmes masculins. Or, des recherches antérieures ont mis en évidence la complexité des vécus et des trajectoires sexuelles des femmes s'identifiant comme lesbiennes ou bisexuelles. Cette complexité tient à la fluidité des identités, des attirances et des comportements sexuels (Chetcuti et al., 2012) ainsi qu'à diverses formes de contraintes à l'hétérosexualité exercées sur les adolescentes et les jeunes femmes (Lebreton, 2014). En ce sens, une intervention adéquate des professionnel.le.s auprès des usagères lesbiennes et bisexuelles devrait prendre en compte que l'orientation sexuelle déclarée ne détermine pas les comportements sexuels de la personne. Ainsi, une femme peut se considérer comme principalement homosexuelle sur le plan des désirs, revendiquer une identité lesbienne et avoir des rapports sexuels parfois ou souvent avec des hommes ou des personnes trans. L'inverse est aussi possible, à savoir que des conduites hétérosexuelles n'excluent pas une remise en question de l'orientation sexuelle. Les trajectoires sexuelles et identitaires ne sont pas fixes ou linéaires au cours d'une vie.

Deuxièmement, la minimisation des risques liés aux ITSS tient au nombre limité de données épidémiologiques disponibles concernant la transmission des ITSS lors de rapports sexuels entre femmes, et ce, sans compter la diversité des corps associés à une identité de genre dans le spectre féminin. S'il est établi que les risques de transmission du VIH sont moindres pour les femmes ayant des rapports sexuels avec d'autres femmes, à l'exception de contacts avec du sang, quelques recherches révèlent des risques significatifs de transmission d'ITSS telles que l'herpès ou le virus du papillome 
humain (VPH) (Marrazzo, Coffey et Bingham, 2005). Or, comme le montre l'enquête de Power, McNair et Carr (2009) réalisée en Australie auprès de 349 femmes - 180 lesbiennes, 32 bisexuelles, 28 queer et 109 hétérosexuelles -, les lesbiennes se sentent moins à risque de contracter le VPH et font moins de tests Pap que les femmes hétérosexuelles. Selon les auteur.e.s, le discours dominant de prévention et de promotion de pratiques sexuelles sécuritaires qui cible principalement les femmes hétérosexuelles et les hommes ayant des relations avec d'autres hommes a une influence sur la perception des lesbiennes quant aux facteurs de risque dans leur propre situation (aucune observation n'est formulée dans le cas des bisexuelles). Ce discours contribue à renforcer leur perception qu'elles sont moins à risque et fait qu'elles seront aussi moins portées à chercher des soins préventifs. Bref, il serait difficile pour elles d'obtenir une information juste, à jour et fiable en matière de santé sexuelle. Dans la région de Montréal d'où proviennent la majorité des répondant.e.s de notre échantillon, il existe davantage de ressources en santé, d'organismes communautaires et de programmes de prévention et de soutien ciblant les hommes gais et les hommes ayant des relations sexuelles avec d'autres hommes (HARSAH). Un plus grand effort d'adaptation des services de santé à l'égard de ces groupes a été déployé dans le cadre des stratégies de lutte contre le $\mathrm{VIH} /$ sida dans les dernières décennies. En comparaison, une recherche réalisée sur l'ensemble du territoire québécois a démontré que peu d'initiatives similaires à l'égard des femmes de minorités sexuelles avaient été réalisées au Québec (Mimeault, 2003). Selon les commentaires émis par les répondantes, la situation ne semble guère s'être améliorée. Ce constat met en lumière l'institutionnalisation du privilège masculin et cisgenre.

Finalement, une troisième hypothèse explicative est l'inconfort de certain.e.s professionnel.le.s des services sociaux et de santé face à la non-conformité de genre des usagères des services. Cette dimension a davantage été rapportée par les femmes de l'étude. En effet, la pression sociale et les normes sociales de genre affectent les lesbiennes et les bisexuelles au même titre que les autres femmes. Cet inconfort de certain.e.s professionnel.le.s témoigne de l'hétéronormativité de nos institutions de santé qui s'appuie sur la conception culturelle essentialiste voulant que la masculinité et la féminité soient des constituantes naturelles indépassables (Bastien Charlebois, 2011). L'épilation, par exemple, est une pratique hautement normative dans les sociétés occidentales modernes (Toerien et Wilkinson, 2004). L'interaction avec des femmes qui refusent de s'épiler suscite parfois chez certain.e.s professionnel.le.s des réactions d'étonnement à minima, de dégoût et de rejet dans les pires situations. Cet inconfort de la part des prestataires de services ne communique pas une attitude positive et ne permet pas l'établissement d'une bonne interaction avec les usagères. Les mauvaises expériences de services ont ultérieurement un impact sur l'accès, le recours aux services et la qualité de la prise en charge. Ainsi, un travail d'information et de déconstruction des représentations auprès des professionnel.le.s des services sociaux et de santé serait nécessaire afin de favoriser l'adoption de stratégies adéquates et d'éviter des maladresses dans l'intervention, qu'il s'agisse d'un accompagnement psychosocial ou de prévention des ITSS.

\section{Les limites de l'étude}

L'insatisfaction des lesbiennes et des bisexuelles à l'égard des services sociaux et de santé s'est exprimée à travers les commentaires et les exemples concrets fournis dans trois sections du questionnaire en ligne. Tout comme pour les résultats quantitatifs (Dumas et al., 2016), l'insatisfaction est plus grande du côté des femmes lesbiennes, bisexuelles, queers et trans que chez les hommes cisgenres gais et bisexuels. Deux limites de l'étude sont à souligner. D'une part, elle se base sur un 
échantillon non représentatif constitué de volontaires recrutés principalement à travers les réseaux associatifs. II est possible que l'invitation à formuler des appréciations et des critiques vis-à-vis du réseau de services sociaux et de santé ait été davantage saisie par les femmes les moins satisfaites et qui n'ont guère d'autres canaux pour faire valoir leur mécontentement. D'autre part, la moyenne d'âge des répondantes $(32,4$ ans, écart-type de 13,9) est nettement inférieure à celle des répondants $(41,5$ ans, écart-type de 17,1). On peut faire l'hypothèse que les répondantes plus jeunes, du moins une partie d'entre elles, adoptent des comportements moins normatifs et sont davantage préoccupées par des questionnements relatifs à leur trajectoire identitaire et à leur santé sexuelle, et que certaines ont exprimé leur frustration de ne pas obtenir de réponses satisfaisantes dans le réseau de la santé et des services sociaux.

\section{Conclusion}

La contribution de cette étude est essentielle puisqu'elle vient pallier le peu d'enquêtes populationnelles sur la santé des minorités sexuelles au Québec prenant en compte le spectre large de la diversité sexuelle et de genre. Elle met en évidence les lacunes de l'offre de services sociosanitaires auprès de la population lesbienne et bisexuelle : présomption de l'hétérosexualité, manque d'établissement d'un climat facilitant l'échange sur les diverses dimensions de l'orientation sexuelle, inadéquation des services de dépistage et de prévention des ITSS, inconfort des professionnel.le.s face à une expression de genre non conforme aux normes hétérosexistes. Cette étude fait également état des pistes d'intervention suggérées par les usagères elles-mêmes, axées principalement sur la formation des professionnel.le.s concernant les enjeux de santé spécifiques aux personnes LGBT et sur une offre de services répondant mieux à leurs besoins. Des recherches futures devraient inclure les expériences de plus en plus diversifiées des personnes dont l'orientation sexuelle et l'identité de genre, et leurs différentes combinaisons, ne se conforment pas aux modèles hétéronormatifs. Par exemple, elles pourraient s'intéresser aux expériences des hommes trans qui n'effectuent pas de modifications à leurs organes génitaux puisque certains de leurs enjeux de santé, entre autres sexuelle et reproductive, sont similaires à ceux d'une femme cisgenre. En somme, les résultats soulignent la nécessité d'une remise en question du carcan de l'hétéronormativité tant dans les pratiques institutionnelles en santé que dans les recherches universitaires.

FEUGÉ, Éric Alain, doctorant et enseignant en psychologie Université du Québec à Montréal

CHAMBERLAND, Line, Ph. D. Sociologie, professeure Département de sexologie Université du Québec à Montréal

KAMGAIN, Olivia, M.A. Administration publique, adjointe de recherche Université du Québec à Montréal

DUMAS, Jean, post-doctorant en communication Université du Québec à Montréal 


\section{RÉFÉRENCES}

Alencar Albuquerque, G. A., C. De Lima Garcia, G. Da Silva Quirino, M. J. Henrique Alves, J. Moreira Belém, F. W. Dos Santos Figueiredo et al. (2016). « Access to health services by lesbian, gay, bisexual, and transgender persons: Systematic literature review », BMC International Health and Human Rights, vol. 16, $\mathrm{n}^{\circ} 2$.

[http://bmcinthealthhumrights.biomedcentral.com/articles/10.1186/s12914-015-0072-9]

Bastien Charlebois, J. (2011). « Au-delà de la phobie de l'homo : quand le concept d'homophobie porte ombrage à la lutte contre l'hétérosexisme et l'hétéronormativité », Reflets, vol. 17, n 1, p. 112 149.

Bauer, G. R., X. Zong, A. I. Scheim, R. Hammond et A. Thind (2015). « Factors impacting transgender patients' discomfort with their family physicians: A respondent-driven sampling study, PLOS ONE, vol. $10, \mathrm{n}^{\circ} 12$, e0145046.

Butler, J. (2006). Trouble dans le genre. Le féminisme et la subversion de l'identité, Paris, La Découverte/Poche.

Chamberland, L. (2006). "La loi 84 - Vers une reconnaissance de l'homoparentalité », Conjonctures, $\mathrm{n}^{\circ} 41 / 42$, p. $43-54$.

Chamberland, L., et E. Saewyc (2011). « Stigmatisation, vulnérabilité et résilience : la santé psychosociale des minorités sexuelles et de genre au Canada », Revue canadienne de santé mentale communautaire, vol. $30, \mathrm{n}^{\circ} 2$, p. 5-11.

Chamberland, L., D. Beaulieu-Prévost, D. Julien, A. N'Bouke et C. de Pierrepont (2012). « Portrait sociodémographique et de santé des populations LGB au Québec : analyse secondaire des données de l'Enquête de santé dans les collectivités canadiennes », rapport de recherche, Montréal, Université du Québec à Montréal.

[http://chairehomophobie.uqam.ca/upload/files/Rapport_Portrait_sociodemo_Chamberland_et_al._R esume_juillet_2012.pdf]

Chetcuti, N., N. Beltzer, N. Methy, C. Laborde, A. Velter, N. Bajos et CSF Group (2013). « Preventive care's forgotten women: Life course, sexuality, and sexual health among homosexually and bisexually active women in France », Journal of Sex Research, vol. 50, n 6, p. 587-597.

DeHart, D. D. (2008). « Breast health behavior among lesbians: The role of health beliefs, heterosexism, and homophobia », Women \& Health, vol. 48, n 4, p. 409-427.

Dumas, J., Chamberland L. et O. Kamgain (2016). « Adéquation des services sociaux et de santé avec les besoins des minorités sexuelles : résultats et recommandations de la recherche-action participative menée au CSSS Jeanne-Mance », rapport de recherche, Chaire de recherche sur l'homophobie, Université du Québec à Montréal, Montréal.

[http://chairehomophobie.uqam.ca/upload/files/Rapport_CSSS.pdf] 
Fish, J. (2006). Heterosexism in Health and Social Care, Basingstoke, Palgrave.

Fredriksen-Goldsen, K., K. Hyun-Jun, S. Barkan, K. Balsam et S. Mincer (2010). «Disparities in health-related quality of life: A comparison of lesbians and bisexual women », American Journal of Public Health, vol. 100, n¹1, p. 2255-2261.

Herek, G. (2004). «Beyond "Homophobia": Thinking about sexual prejudice and stigma in the twentyfirst century », Sexuality Research \& Social Policy, vol. 1, n² 2, p. 6-24.

Lebreton, C. (2014). Rapports sociaux de sexe et sexualité dans le Québec contemporain : les trajectoires adolescentes lesbiennes, thèse de doctorat en sociologie, Université du Québec à Montréal, Montréal.

Marrazzo, J., P. Coffey et A. Bingham (2005). « Sexual practices, risk perception and knowledge of sexually transmitted disease risk among lesbian and bisexual women », Perspectives on Sexual and Reproductive Health, vol. 37, nº 1, p. 6-12.

Mimeault, I. (2003). Pour le dire... Rendre les services sociaux et les services de santé accessibles aux lesbiennes, rapport de recherche, Montréal, Réseau québécois d'action pour la santé des femmes. [http://rqasf.qc.ca/files/sante-lesbiennes.pdf]

Power, J., R. McNair et S. Carr (2009). « Absent sexual scripts: Lesbian and bisexual women's knowledge, attitudes and action regarding safer sex and sexual health information ", Culture, Health \& Sexuality, vol. 11, $\mathrm{n}^{\circ} 1$, p. 67-81.

Simoni, J., L. Smith, K. Oost, K. Lehavot et K. Fredriksen-Goldsen (2017). « Disparities in physical health conditions among lesbian and bisexual women: A systematic review of population-based studies », Journal of Homosexuality, vol. 64, n 1, p. 32-44.

Steele, L., J. Tinmouth et A. Lu (2006). « Regular health care use by lesbians: A path analysis of predictive factors », Family Practice, vol. 23, n 6, p. 631-636.

Tjepkema, M. (2008). Utilisation des services de santé par les gais, les lesbiennes et les bisexuels au Canada, Rapports sur la santé au catalogue de Statistique Canada, Statistique Canada.

[http://www.statcan.gc.ca/pub/82-003-x/2008001/article/10532-fra.pdf]

Toerien M. et S. Wilkinson (2004). « Exploring the depilation norm: A qualitative questionnaire study of women's body hair removal », Qualitative Research in Psychology, vol. 1, nº 1, p. 69-92. 